\title{
TRANSFERS OF MEANING
}

\author{
Geoffrey Nunberg \\ Xerox Palo Alto Research Center and Stanford University \\ Xerox PARC \\ 3333 Coyote Hill Road \\ Palo Alto CA, 94304 USA \\ Internet: nunberg@parc.xerox.com
}

\begin{abstract}
In one form or another, the phenomena associated with "meaning transfer" have become central issues in a lot of recent work on semantics. Speaking very roughly, we can partition approaches to the phenomenon along two dimensions, which yield four basic points of departure. In the first two, people have considered transfer in basically semantic or linguistic terms. Some have concentrated on what we might call the paradigmatic aspects of transfer, focusing on the productive lexical processes that map semantic features into features - for example, the "grinding" rule that applies to turn the names of animals into mass terms denoting their meat or fur. This the approach that's involved in most recent work on "regular polysemy," "systematic polysemy," and the like, for example by Apresjan, Ostler and Atkins, Briscoe and Copestake, Nunberg and $\mathrm{Za}$ enen, Wilensky, Kilgarriff and a number of other people. Other people have emphasized the syncategorematic aspects of transfer; that is, the ways meaning shifts and specifications are coerced in the course of semantic composition. This is an approach that hass been developed in particular by James Pustejovsky and his collaborators, building on earlier work on type shifting.
\end{abstract}

As opposed to these, there are conceptual and pragmatic approaches to transfer, which focus on the extralinguistic circumstances that license transfers of various types. Here again there are both paradigmatic and syncategorematic approaches, loosely speaking. The first is exemplified in a lot of recent work on metaphor by people associated with the "cognitive linguistics" school, which has focused chiefly on the relations between domains of experience that metaphor variously exploits and imputes. The second is represented by work on indirect speech within Gricean pragmatics, Relevance Theory, and the like, which has been chiefly concerned with specifying the conversational conditions that give rise to metaphor, irony, and analogous phenomena.
Of course this categorization is somewhat factitious. The borders between these approaches are highly porous, and most work on transfer overlaps several of them. This is entirely appropriate, since these are in no sense competing theories or accounts of the phenomena. Transfer is clearly a linguistic process, and in many of its most important forms a lexical one. But it just as clearly has its basis in very general cognitive and communicative principles. And while it's reasonable that people should choose to focus on one or another of these considerations relative to their immediate interests, it is also useful to keep the Big Picture in mind, lest we inadvertently ascribe to one domain of explanation a responsibility that more properly belongs to another. This is the picture I want to sketch out in this talk.

A comprehensive account of transfer has to make appeal to three different kinds of regularities or rules. The first are nonlinguistic: the correspondences between domains, real or imputed, that transfer invokes, and the communicative interests that may make these invocations useful or instructive - they enable us to identify one thing in virtue of its relation to another, explain an abstract domain by reference to a concrete one, and so forth. Second, there is the repertory of general linguistic processes of transfer that exploit these correspondences and principles. By these I have in mind not traditional categories like metaphor, synecdoche, and metonymy - distinctions that have basically to do with the kinds of domain correspondences that transfer exploits - but the various types of operations that make possible typeshifting and sortal reassignment of expressions, syntactic recategorizations, and deferred indexical reference. These processes may cross-cut the types of domain correspondences that they exploit, and I'll show that we often find a single type of domain correspondence underlying two or more distinct semantic processes of transfer. Third, there are the language-specific instantiations of these operations, for example in the form of constructions or lexical rules that license particular types or 
subtypes of transfers (for example some language, like Greenlandic Eskimo, permit "grinding" of tree names to yield names of types of woods, but not of animal names to yield the names of furs or meats.)

In the first part of this talk, I'll focus on one of the general processes that underlie transfer: the semantic operation of "predicate transfer," which licenses the sortal reassignment of expressions denoting properties and relations, and which underlies a great deal of lexical polysemy. The process I have in mind is illustrated by an example like "I am parked out back." This is usually regarded as a classic instance of metonymy - i.e., "person" for "car" - where we use an expression that would conventionally denote one thing to refer to some other thing to which it is connected by a "relation of contiguity." But I'll show that on consideration there are compelling reasons for supposing that $I$ here refers to the speaker, rather than his car. (For one thing the number of the pronoun doesn't vary according to the number of cars involved: if you had two cars parked out back you wouldn't say "We are parked out back," though of course this would be the appropriate thing to say to refer to a single car owned by two or more people). And other morphological and syntactic observations support the same conclusion: in examples like this what has been transferred is the meaning of the predicate, rather than its argument. That is, the predicate parked out back has a transferred reading here: it denotes the property that the speaker acquires in virtue of his relation to a car that has the property of being parked out back.

Two conditions have to be satisfied before predicate transfers like this one are licensed. First, there has to be a salient correspondence (more specifically, an injective function) between the properties of things in one domain and the properties of things in another; e.g., between the locations of cars in a lot and the properties that distinguish the owner of one car from the owner of another. Second, it has to be either useful or interesting to know that these acquired or inherited properties apply to their carriers: that's why we can say "I am parked out back" to someone who is about to go get the car, whereas it is hard to imagine a context in which one would want to say "I was once driven by Ricardo Montalban."

I will give a simple formal account of these conditions on predicate transfer, and then show how it resolves some familiar syntactic and semantic difficulties. Take Jackendoff's example, "Ringo squeezed himself into a narrow parking space." If we analyze this as involving a metonymy, we will have to say that the reflexive here denotes something distinct from its antecedent, and so make provision for certain sortal shifts in giving the identity conditions on reflexivization and other rules and constructions ordinarily require coreference of pronoun and antecedent. Whereas now we will take squeeze into a narrow parking place as a transferred predicate that denotes a relation between persons: in virtue of having squeezed his car into a space, that is, Ringo has also done something noteworthy to himself. More generally, I'll argue that the conditions on rules of anaphora and similar operations need never provide for sortal shifts; sortally speaking, we must always take "syntactic identity" in the strictest possible way.

In the second part of this paper, I'll show how predicate transfer is instantiated lexically in the rules that provide for systematic polysemy. I'll mention several familiar cases: grinding, conversion of names of artists to the names of their works (e.g., a Picasso, an Agatha Christie, and the use of the names of publications like newspaper and magazine for the organizations that produce them. Each of these processes is subject to a variety of constraints, which may answer any of several different principles. Some are due to the absence of perceived domain correspondences of the appropriate type (for example, the reluctance of words like mammal and bird to undergo grinding. Some are explained by the fact that the acquired property denoted by the transferred predicate is insufficiently noteworthy or criterial: that is why we don't say She was reading a Kafka. Still others are due to the absence of specific lexical licenses for certain types of transfer; this explains why we don't generally use the "artist for work" rule to derive the names of musical works (?two Beethovens, ?several Elvises), or why grinding does not apply in English to derive the names of liquids? We always cook with olive. All of this by way of showing why it is important to bear in mind the heterogeneity of the mechanisms that underlie transfers of all types. 